\title{
CORRIGENDUM
}

\section{The Need for Digital Health Solutions in Deep Brain Stimulation for Parkinson's Disease in the Time of COVID-19 and Beyond}

In Rammo et al. (1), Figs. 1 and 2 were published with watermarks. The correct figure images are provided below.

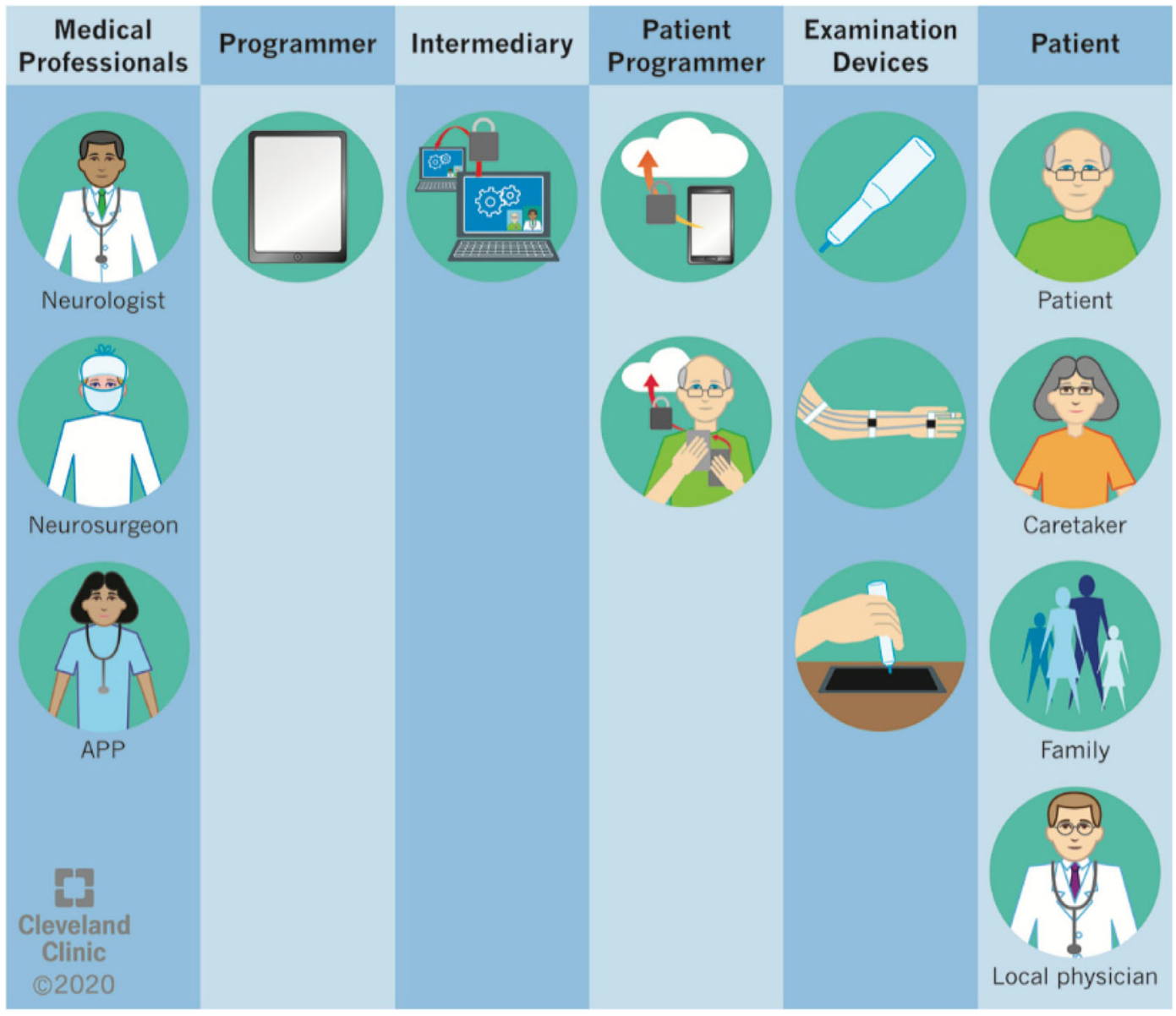

Figure 1. The necessary components of a distance health encounter with the medical professional on the left as it transitions to the patient on the right and vice-versa. [Color figure can be viewed at wileyonlinelibrary.com]. [Color figure can be viewed at wileyonlinelibrary.com] 


\section{Synchronous}
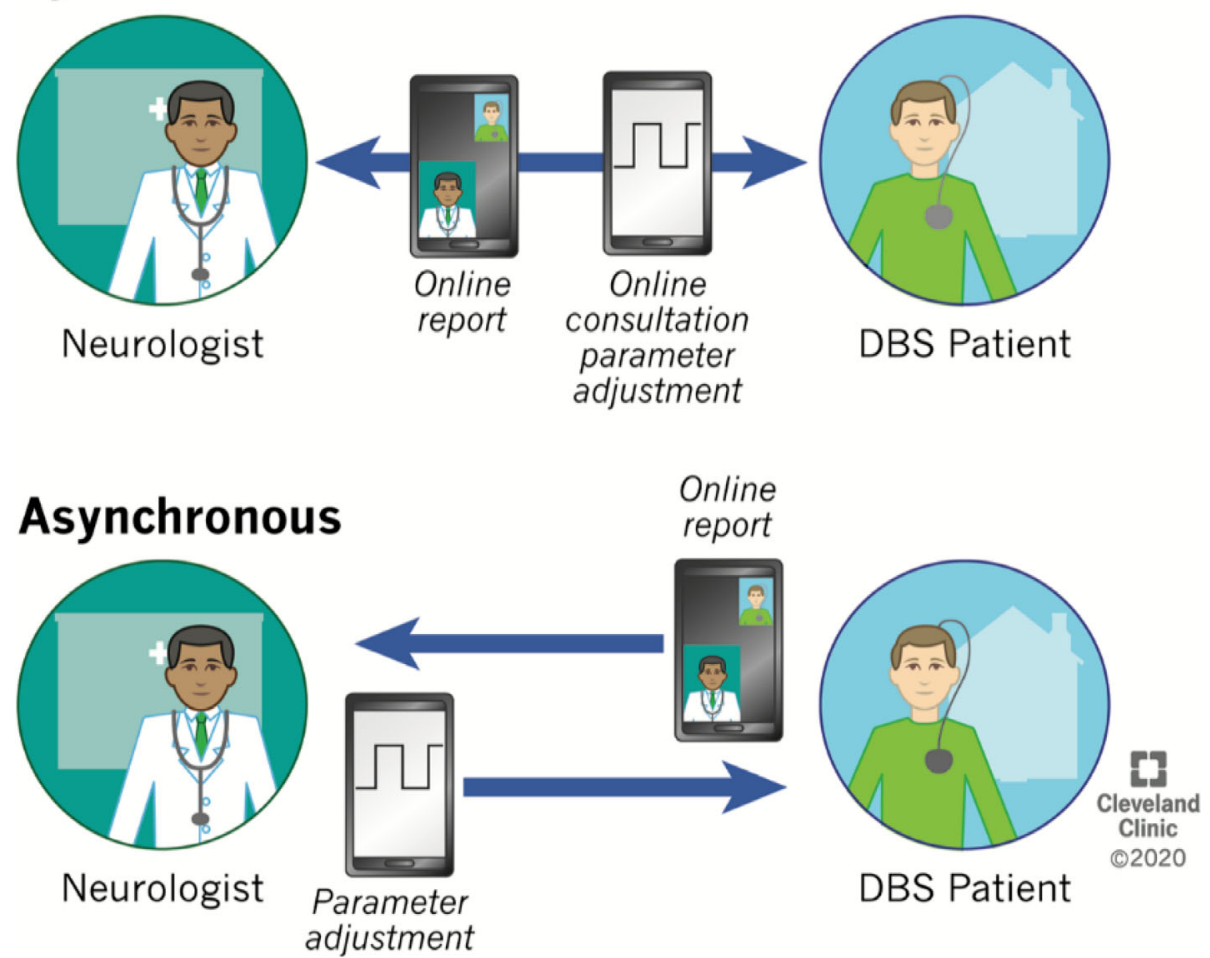

Neurologist adjustment

Figure 2. The implementation of a digital health encounter between a patient and his or her neurologist in synchronous (top) and asynchronous (bottom) paradigms. [Color figure can be viewed at wileyonlinelibrary.com]. [Color figure can be viewed at wileyonlinelibrary.com]

The online version has been corrected.

We apologize for this error.

\section{REFERENCE}

1. Rammo R, Gostkowski M, Rasmussen PA, Nagel S, Machado A. The need for digital health solutions in deep brain stimulation for Parkinson's Disease in the time of COVID-19 and beyond. Neuromodulation 2021;24:331-336. 